\title{
Development of infrastructure and logistics of a field hospital created to delivering care during dengue outbreak
}

\author{
José Frederico Corte ${ }^{1 *}$, Artur Martins Codeço ${ }^{1}$ and Cléber de Moraes Motta ${ }^{2}$
}

\begin{abstract}
Dengue is considered one of the main tropical diseases in the world and Brazil is among the countries susceptible to some infectious diseases. Thus, it is necessary to provide medical care in difficult-to-access regions with limited resources in a quick and effective way. To achieve this goal, mobile health structures, with versatile characteristics, appear to be an alternative to safely assist in emergencies in locations that need such action. In the first 10 weeks of 2015, the São Paulo City Hall confirmed 4,436 dengue cases among 15,789 reported ones and the Hospital Israelita Albert Einstein (HIAE), in partnership with local authorities, volunteered to deliver multidisciplinary care to patients with dengue. This study describes the logistic, resource, and infrastructure efforts deployed in a short time frame for in loco care delivered to communities affected by the dengue outbreak. Despite the care and logistical planning of the mobile hospital, several unexpected and challenging situations appeared during the campaign. However, all challenges were overcome through teamwork. Teams maintained their commitment with delivering high quality care.
\end{abstract}

Keywords: Field hospital, Epidemy, Dengue fever

\section{Background}

Dengue is considered one of the major tropical diseases in the world. This disease is caused by Flavivirus and transmitted to humans by infected Aedes aegypti mosquitoes [1]. Early diagnosis followed by immediate and adequate treatment has a positive impact on the prognosis [1-3]. Because Brazil is among the countries susceptible to some infectious diseases, it must provide quick and effective medical care in difficultto-access regions with limited resources. Hence, mobile health structures, with versatile characteristics, appear to be an alternative to safely assist in emergencies in locations that need such action. During the first 10 weeks of 2015, the São Paulo City Hall confirmed 4,436 dengue cases among 15,789 reported ones, a number three times greater than recorded in the same period for 2014 [4]. The Hospital Israelita Albert Einstein (HIAE), aware of its position and its social responsibility in facing this challenge, and in partnership with local authorities, volunteered to deliver multidisciplinary

\footnotetext{
* Correspondence: jose.corte@einstein.br

${ }^{1}$ Resident in Healthcare Administration at Sociedade Beneficente Israelita Brasileira Albert Einstein, São Paulo, Brazil

Full list of author information is available at the end of the article
}

care to patients with dengue. This study describes the logistic, resource, and infrastructure efforts deployed in a short time frame for in loco care delivered to communities affected by the dengue outbreak. The campaign against dengue in the hospital focused on two main fronts: care and logistics. The first included a clinical screening environment of urgency and emergency, assessment by a multidisciplinary team, collection and analysis of laboratory samples, clinical observation and therapeutic management. The team included physicians, nurses, pharmacists, and laboratory technicians. The second front promoted activities to support electricity generation, a basic sanitation system, nutritional support for patients and professionals, communication (a press office and efforts to establish and maintain the relationship with basic health units and referral hospitals to which patients were transferred), transportation of equipment, materials, and people; control, storage, and transfer of generated information; control and biomedical waste; human resource management; safety measures and research and support for areas such as a nutrition, security, medical records and clinical engineering services. Care for patients began with screening to exclude cases that did not fulfill criteria for 
dengue suspicion [5]. Patients likely to be infected were referred for collection of vital signs and laboratory tests (complete blood count and serological tests). After this stage, patients with blood count results were referred to a medical visit; at this time, the following possible therapies were defined: transfer of the patient to a basic health unit (patients with conditions other than dengue), discharge with therapeutic guidance, discharge with request for a return visit within 24-48 h, clinical observation of the patient in a tent and administration of hydration therapy, or stabilization followed by transfer to a high-complexity service (direct contact for referral) [5]. To meet the care expectations proposed, professionals were selected and recruited voluntarily at HIAE, followed by training for development of care teams, technical teams and support teams. The training programs respected local characteristics and sought standardization of information, operations, processes and actions for rapid, efficient and safe responses, accordingly integrated with members who were involved in operations. To assist the population, we made available two $200 \mathrm{~m} 2$ tents (in total) that covered a screening area and initial clinical evaluation area (location for waiting and care delivery by the nursing team), a location devoted to adult care and one devoted to pediatric care, a specific sector for medication/hydration composed of 20 chairs and one bed, an emergency room with materials recommended by Advanced Cardiovascular Life Support for adult/pediatric care, and one ambulance for transfer. The laboratory, structured within the basic health unit, was equipped with a device to perform a complete blood count test with release in 40 to $60 \mathrm{~s}$. In addition, two laboratory technicians also did quick tests for dengue, with diagnosis within $15 \mathrm{~min}$. Results were attached to the patient's medical record and forwarded to the physician for evaluation. To assist the laboratory in case of problems with equipment, the clinical engineering team was requested to give priority to any maintenance need during the campaign. Medicine selection was based on the care protocol of the Brazil Ministry of Health [5], which primarily indicates hydration solutions, antipyretics and analgesics. Medicines were stocked according to Brazilian Health Surveillance Agency (ANVISA, acronym in Portuguese) in two different places: a central storehouse supplied by HIAE, and a stock area at the basic health unit (inside the tent) that was refilled daily or according to necessity. The nutrition team ensured nutritional support for patients who were being assisted at the mobile hospital as well as collaborators who were working on the campaign. The Department of Information Technology offered support by storing data generated during patient encounters. These data were fundamental for providing support statistics and information for future studies. Patients' medical records were housed in the Medical Records and Health Statistics Services. Anther instrument used during this action was the telemedicine technology that, along with HIAE team, enabled the evaluation of more complex cases so that the best guidance for therapeutic management could be provided. Although care occurred during a period of $12 \mathrm{~h}$ each day, a security team was hired to protect the area in order to ensure that the hospital's tents would not be harmed. In addition, to assist those working in the campaign, we provided insect repellent, air conditioners in tents and transportation to the basic health unit.

\section{Conclusion}

Despite the care and logistical planning of the mobile hospital, several unexpected and challenging situations appeared during the campaign. However, all challenges were overcome through teamwork. Teams maintained their commitment with delivering high quality care.

\section{Competing interests}

The authors declare that they have no competing interests.

Authors' contributions

All authors read and approved the final manuscript.

\section{Author details}

${ }^{1}$ Resident in Healthcare Administration at Sociedade Beneficente Israelita Brasileira Albert Einstein, São Paulo, Brazil. ${ }^{2}$ MD, Preceptor of Healthcare Administration Residency Program at Sociedade Beneficente Israelita Brasileira Albert Einstein, São Paulo, Brazil.

Received: 17 April 2015 Accepted: 12 May 2015

Published online: 01 September 2015

\section{References}

1. Teixeira MG, Barreto ML. Diagnosis and management of dengue. BMJ. 2009;339:1189-93.

2. Teixeira MG, Costa MCN, Barreto F, Barreto ML. Dengue: twenty-five years since reemerge in Brazil. Cad Saude Publica. 2009;25(suppl 1):S7-S18.

3. Marra AR, de Matos GFJ, Janeri RD, Machado PS, Schvartsman C, dos Santos OFP. Managing patients with dengue fever during na epidemic: the importance of a hydratation tent and of a multidisciplinar approach. BMC Research Notes. 2011;4:335.

4. Prefeitura Municipal de São Paulo. Situação da transmissão de dengue no município de São Paulo. Disponível em: http://www.capital.sp.gov.br/static/ 2015/03/vfFZd7bfsvNv9mqaoY1Oww.pdf. Acesso em: 02 de abril de 2015.

5. Brasil. Ministério da Saúde. Secretaria de Vigilância em Saúde. Diretoria Técnica de Gestão. Dengue: diagnóstico e manejo clínico: adulto e criança / Ministério da Saúde, Secretaria de Vigilância em Saúde, Diretoria Técnica de Gestão. - 4. ed. - Brasília: Ministério da Saúde, 2013. 80 p.

\section{Submit your next manuscript to BioMed Central and take full advantage of:}

- Convenient online submission

- Thorough peer review

- No space constraints or color figure charges

- Immediate publication on acceptance

- Inclusion in PubMed, CAS, Scopus and Google Scholar

- Research which is freely available for redistribution 